\title{
É mesmo (só) Transtorno de Déficit de Atenção/Hiperatividade (TDAH)? Avaliando TDAH e encontrando dislexia
}

\author{
Marilene Tavares Cortez ${ }^{1}$ \\ Luciana Karine de Souza ${ }^{2}$ \\ Ângela Maria Vieira Pinheiro ${ }^{3}$ \\ ${ }^{1}$ Universidade Federal de Minas Gerais, $M G$, Brasil \\ http://orcid.org/0000-0003-3059-0219 \\ ${ }^{2}$ Universidade Federal do Rio Grande do Sul, RS, Brasil \\ Universidade Federal de Minas Gerais, MG, Brasil \\ http://orcid.org/0000-0001-9641-6163 \\ ${ }^{3}$ Universidade Federal de Minas Gerais, MG, Brasil \\ http://orcid.org/0000-0002-5852-4320
}

\begin{abstract}
Resumo
Dado que déficits na velocidade de processamento são fator de risco para TDAH e dislexia, investigou-se o perfil cognitivo de indivíduos com TDAH e com indicadores de dislexia. Compuseram a amostra escolares com idade entre 7 e 15 anos (62\% masculino), sendo 23 diagnosticados com TDAH, 17 em risco para TDAH e 31 crianças em grupo controle. A avaliação incluiu os subtestes do WISC e tarefas tais como Stroop Palavra-Cor, nomeação seriada rápida, fluência verbal, repetição de pseudopalavras, e supressão de fonemas. Após, os participantes foram reclassificados: 45 sem TDAH nem risco para dislexia; 9 com TDAH; 6 com sinais de dislexia; e 11 com TDAH e sinais de dislexia. Os sérios déficits cognitivos apresentados pelo último grupo confirmam os achados sobre o seu fracasso escolar. A comorbidade TDAH-dislexia necessita ser melhor investigada na avaliação diagnóstica de escolares com queixas de dificuldades de aprendizagem.
\end{abstract}

Palavras-chave: processos cognitivos, avaliação, transtorno da falta de atenção com hiperatividade, dislexia.

\section{Is it really (just) Attention Deficit-Hyperactivity Disorder (ADHD)? Assessing ADHD and finding dyslexia}

\begin{abstract}
Given that processing speed deficits are a risk factor for ADHD and dyslexia, we investigated the cognitive profile of individuals with ADHD and with indicators of dyslexia. The sample comprised children between the ages of 7 and 15 (62\% male), 23 of whom were diagnosed with ADHD, 17 were at risk for ADHD, and 31 formed the control group. The evaluation included WISC subtests and tasks such as Word-Color Stroop, rapid automatized naming, verbal fluency, pseudowords repetition, and phoneme suppression. After this procedure, the participants were reclassified: 45 without ADHD or signs of dyslexia; 9 with ADHD; 6 with signs of dyslexia; and 11 with ADHD and signs of dyslexia. The serious cognitive deficits presented by the latter justify the findings on school failure to which it is subject. ADHDdyslexia comorbidity needs to be better investigated in the diagnostic evaluation of schoolchildren with complaints of learning difficulties.
\end{abstract}

Keywords: cognitive processes, assessment, attention deficit disorder with hyperactivity, dyslexia. 


\title{
Es mismo (sólo) Transtorno de Déficit de Atención/Hiperatividad (TDAH)? Avaliando TDAH y encontrando dislexia
}

\begin{abstract}
Resumen
Dado que los déficits en la velocidad de procesamiento son factor de riesgo para TDAH y dislexia, se investigó el perfil cognitivo de individuos con TDAH y con indicadores de dislexia. Compusieron la muestra escolares con edad entre 7 y 15 años (62\% masculino), siendo 23 diagnosticados con TDAH, 17 en riesgo para el TDAH y 31 para grupo controle. La evaluación incluyó las subpruebas del WISC y tareas tales como Stroop Palabra-Color, nombramiento seriado rápido, fluencia verbal, repetición de pseudopalabras, y supresión de fonemas. Después de ese procedimiento los participantes fueron reclasificados: 45 sin TDAH ni signos de dislexia; 9 con TDAH; 6 con signos de dislexia; y 11 con TDAH y signos de dislexia. Los serios déficits cognitivos presentados por el último grupo confirman los hallazgos sobre su fracaso escolar. La comorbilidad TDAH-dislexia necesita ser mejor investigada en la evaluación diagnóstica de escolares con quejas de dificultades de aprendizaje.
\end{abstract}

Palabras clave: procesos cognitivos, evaluación, trastorno por déficit de atención con hiperactividad, dislexia.

No DSM-5 $5^{\mathrm{TM}}$, o Transtorno de Déficit de Atenção/ Hiperatividade (TDAH) passou a ser considerado um transtorno do neurodesenvolvimento, separado das psicopatologias agrupadas como alterações de conduta (American Psychiatric Association [APA], 2014; Snowling \& Hulme, 2012; Tannock, 2013). De acordo com o manual, "indivíduos com o TDAH podem exibir problemas cognitivos em testes de atenção, função executiva ou memória" (APA, p. 61), o que, na prática, significa que essas funções devem ser avaliadas em um procedimento para o diagnóstico da condição. $\mathrm{O}$ reconhecimento de "problemas cognitivos" no TDAH representa um avanço, uma vez que as escalas comportamentais, largamente empregadas para avaliar o indivíduo com esse transtorno, "podem gerar respostas tendenciosas, tanto para um padrão de normalidade (falso-negativo), quanto para um patológico (falso-positivo)" (Abujadi \& Moraes, 2010, p. 142).

Pennington (2006) propôs um modelo de múltiplos déficits cognitivos envolvidos no TDAH. Em seus pressupostos, preconiza que: (a) a etiologia do TDAH é multifatorial, envolvendo a interação de fatores protetivos, genéticos, ambientais e de risco; (b) a presença ou ausência desses fatores altera o desenvolvimento do funcionamento cognitivo; (c) é necessária a identificação de mais de um fator etiológico; (d) comorbidades são previstas, uma vez que a etiologia e os fatores de risco são partilhados; e (e) os fatores responsáveis pela pre- valência dos transtornos ocorrem de forma contínua e quantitativa, em vez de discreta e categórica, "de modo que o limiar que determina a presença do distúrbio é, de certo modo, arbitrário" (Pennington, 2006, p. 404, tradução nossa). Tomados em conjunto, o modelo de múltiplos déficits cognitivos, a nova classificação como transtorno do neurodesenvolvimento e a literatura sobre o funcionamento cognitivo no TDAH colaboram para convocar procedimentos renovados por parte de médicos e psicólogos face a demanda por diagnóstico frente às dificuldades de ajustamento na infância.

Um exemplo da complexidade diagnóstica diante da suspeita de TDAH é a comorbidade com outros transtornos do desenvolvimento. Pesquisas têm mostrado que déficits na velocidade de processamento (VP) é tanto um fator de risco para TDAH como para dislexia (McGrath et al., 2011; Nikolas \& Nigg, 2013; Pennington, 2006). A revisão sistemática de estudos empíricos publicados entre 2000 e 2012, de Prando et al. (2013), mostrou que quase metade das pesquisas sobre a avaliação da linguagem em casos de TDAH identificou a dislexia como comorbidade mais prevalente.

Nessa direção, McGrath et al. (2011, p. 555, tradução nossa) argumentam que o modelo de múltiplos déficits cognitivos é adequado porque "sustenta que os transtornos neurodesenvolvimentais são produzidos por uma combinação de déficits específicos e compartilhados, sendo os compartilhados responsáveis pela comorbida- 
de entre os transtornos". Buscando déficits comuns ao TDAH e à dislexia, os autores identificaram, por meio de análise de equações estruturais, que um déficit na VP é preditor para ambos. Mais especificamente, os preditores para a dislexia foram os déficits na consciência fonológica e na velocidade de nomeação (VN); já o preditor para o TDAH foi um déficit no controle inibitório nas dimensões desatenta e impulsivo-hiperativa do transtorno (McGrath et al., 2011).

O caráter multifacetado do TDAH acarreta implicações diretas quando se busca traçar o perfil cognitivo dessa condição e realizar o seu diagnóstico diante de seus indicadores. Trata-se de uma tarefa complexa que demanda não só a consideração dos critérios do DSM e a avaliação criteriosa das funções cognitivas apontadas, como também a atenção a variáveis psicossociais associadas ao distúrbio (Larroca \& Domingos, 2012; Tannock, 2013).

Com a finalidade de contribuir com o corpo de conhecimentos sobre o funcionamento cognitivo no TDAH, o presente estudo testa um conjunto de medidas para avaliar esse funcionamento em escolares do Ensino Fundamental com o diagnóstico de TDAH. Com base no modelo de Pennington (2006), buscou-se investigar o perfil cognitivo desses indivíduos e possíveis indicadores de dislexia. Como hipóteses, estabeleceu-se que: os escolares com TDAH apresentarão pior desempenho em termos de MT, VP, VN, controle inibitório e atenção, na comparação com alunos sem TDAH; e a avaliação será capaz de detectar alunos com indicadores de dislexia, inclusive em comorbidade com o TDAH.

\section{Método}

\section{Participantes}

Participaram 135 alunos regularmente matriculados no Ensino Fundamental de escolas públicas e privadas da cidade de Divinópolis (Minas Gerais). Segundo os professores e equipe pedagógica da escola, todos os alunos eram alfabetizados e capazes de reconhecer cores e números. Os alunos foram distribuídos em três grupos: 43 com diagnóstico prévio de TDAH, $35 \mathrm{em}$ risco para TDAH, e 57 sem indicadores de transtornos ou síndromes, deficiência sensorial e intelectual, e/ou vítima de violência doméstica. O critério para a formação do grupo com diagnóstico prévio de TDAH foi a emissão prévia de um diagnóstico formal por parte de médico ou psicólogo, solicitado pela escola ou pelos pais, anteriormente à presente pesquisa. Os critérios para a formação do grupo em risco para TDAH e do grupo sem indicadores de transtornos/deficiências foram: o resultado da avaliação de seus professores pelo instrumento SNAPIV (Swanson, Nolan and Pelham Teacher and Parent Rating Scale - SNAP-IV, versão brasileira de Mattos, Serra-Pinheiro, Rohde, \& Pinto, 2006); o resultado do SNAP-IV preenchido pelos pais; e uma anamnese com os pais, que incluiu características psicossociais e de saúde do(a) escolar. Essa anamnese buscou identificar transtornos ou síndromes, deficiência sensorial e intelectual e indicadores de violência doméstica. Os pais também preencheram o Critério de Classificação Econômica Brasil (Associação Brasileira de Empresas de Pesquisa, 2013). O SNAP-IV é uma escala composta de 18 perguntas que investigam aspectos de desatenção (perguntas de 1 a 9) e de hiperatividade e impulsividade (10 a 18), baseadas na categorização do DSM para esses estados e auxiliam na identificação da frequência e gravidade dos sintomas do TDAH. Emprega escores quantitativos em uma escala de 4 pontos: nem um pouco, só um pouco, bastante e demais. O ponto de corte para inclusão no grupo de criança com TDAH foi de mínimo de 6 pontos com as opções de "bastante e demais" nos critérios para desatenção, e igualmente para hiperatividade e impulsividade.

Para confirmar que se tratava de alunos sem rebaixamento intelectual e leitores, todos foram submetidos ao teste Matrizes Progressivas Coloridas de Raven ou ao Matrizes Progressivas Standard de Raven, conforme a idade, e à leitura da fábula "A coruja e a águia", de Monteiro Lobato, sugerida pelos professores. Essa fábula possui 189 palavras, dispostas em 17 frases: 1 
exclamativa, 3 interrogativas e as demais, declarativas. Ao final, 64 alunos foram excluídos da amostra com base em um ou mais dos critérios referidos, bem como mudança de escola ou desistência dos pais.

Portanto, a amostra final do estudo foi de 71 escolares (62\% do sexo masculino), dos quais 23 com diagnóstico prévio de TDAH, 17 em risco para TDAH e 31 crianças alocadas como grupo controle (GC), esses dois últimos grupos determinados conforme os resultados obtidos com o SNAP-IV e a anamnese. A idade variou de 7 a 15 anos, com média de 10 anos e 3 meses. Esses 71 escolares foram submetidos a um processo de reavaliação para a confirmação do status "TDAH", "risco para TDAH" e GC. Essa reavaliação consistiu da aplicação de instrumentos e tarefas para a identificação dos processos cognitivos preservados e deficitários no TDAH, e no TDAH e comorbidades, conforme a literatura.

\section{Instrumentos}

A seguir, são listadas as tarefas e instrumentos aplicados nos participantes. A escolha por essas ferramentas foi fundamentada nos estudos referidos na introdução, os quais relatam déficits de atenção, controle inibitório e MT (APA, 2014), e no elevado índice de comorbidade entre TDAH e dislexia, com déficits reconhecidos na VP e na VN (McGrath et al., 2011; Nikolas \& Nigg, 2013; Pennington, 2006; Prando et al., 2013). Outros estudos também são citados para fundamentar a escolha dos instrumentos.

Subtestes Código, Procurar Símbolos, Dígitos e Aritmética do WISC-III (Wechsler, 1991). Conforme o manual, em todos os subtestes os pontos brutos são transformados em pontos ponderados (máximo de 19), com escores ajustados para uma escala média de 10 pontos e desvio-padrão (DP) igual a 3. O ponto de corte para inclusão no GC (para todas as idades) é de 10. As normas foram estabelecidas para faixas etárias da amostra $(7 ; 8-9 ; 10-11 ; 12-13 ; 14-15$ anos). As análises não apresentaram diferenças estatisticamente significativas nas variâncias e médias das escalas por faixa etária, justificando a elaboração de tabelas únicas em QI para todas as idades. A ponderação dos escores das escalas em QI e índices fatoriais deu-se transformando-os em uma escala de média 100 e DP 15, correspondendo a valores teóricos para uma distribuição normal. Interpretações qualitativas apresentadas pelo WISC-III permitiram estabelecer que a média da população geral é de 90-109 (QI) para a amostra pesquisada. Estudos realizados pelo WISC-III fornecem evidência da validade dos critérios apresentados por ele para distinguir grupos de crianças com transtorno de aprendizagem e com o TDAH. A pesquisa brasileira de Lopes, Farina, Wendt, Esteves e Argimon (2012) evidencia o pior desempenho da criança com o TDAH nos subtestes do WISC-III e no resultado geral de QI. Quatro desses subtestes, bem como os índices de VP e de resistência à distração (RD), foram aplicados/ calculados para o presente estudo, todos utilizados por Lopes et al. (2012) para identificar crianças com TDAH.

O Subteste Código. Avalia a habilidade de decodificar símbolos visualmente, atenção sustentada e seletiva, memória incidental (episódica), componentes da organização perceptiva e persistência motora. Solicita-se que o participante copie símbolos simples que são pareados com formas geométricas também simples (Forma A, 59 símbolos, para 6 e 7 anos de idade) ou com números (Forma B, 119 símbolos, para 8 a 16 anos). Na Forma A, a criança é instruída a preencher as formas geométricas com seus símbolos correspondes. Já na Forma B, a criança deve desenhar um sinal específico embaixo do número correspondente.

O Subteste Procurar Símbolos. Avalia os processos relacionados à atenção, memória e concentração para processar rapidamente a informação visual. Possui Parte A, com 45 símbolos (para idades de 6 e 7 anos) e Parte B, com 45 símbolos (8 a 16 anos). O examinando deve observar símbolos à esquerda e à direita da folha e marcar "sim" caso identifique que o símbolo da esquerda (grupo padrão) aparece no grupo da direita (grupo procura); ou marcar "não", caso contrário. No grupo padrão da Parte $\mathrm{A}$, há apenas 1 símbolo; no grupo da Parte $\mathrm{B}$, há dois símbolos. O escore máximo é de 45 pontos brutos, 
transformados em ponderados, para cada Parte, correspondendo ao número de respostas corretas. Itens que a criança não fez não são incluídos no cálculo do escore.

O Subteste Dígitos. É uma medida que avalia MT, a atenção (Figueiredo \& Nascimento, 2007) e a memória fonológica (Cardoso-Martins, Vítor, \& Navas, 2010). Em relação à memória de trabalho, avalia a capacidade de armazenamento de curto prazo, recordação, repetição imediata, rapidez e span (número de itens que podem ser lembrados com correção). Esse subteste é composto por duas tarefas: Ordem Direta (OD) e Ordem Inversa (OI). A OD relaciona-se ao circuito fonológico "uma vez que a sua realização envolve apenas a armazenagem passiva e temporária de material baseado na fala, com pouca demanda do sistema do executivo central" (Figueiredo \& Nascimento, 2007, p. 314). Já a OI relaciona-se com o executivo central, portanto, envolve distribuir atenção, selecionar estratégias e coordenar diferentes processos. É esperado que o desempenho na OD seja melhor que o da OI. A OD consiste em uma série de oito itens de números em uma sequência aleatória, variando de dois a nove números apresentados de forma crescente. A OI baseia-se em uma série de sete itens, cada um composto de uma sequência aleatória de números, variando de dois a oito números apresentados de forma crescente. Nesta tarefa há uma sequência de dois números ( 8 e 2) como item de treino. $O$ participante deve repetir cada sequência respeitando a ordem em que os números são apresentados nas duas situações: na OD, a ordem original, da esquerda para a direita, é mantida e, na ordem inversa, os números devem ser recuperados da direita para a esquerda. A criança é instruída a ouvir uma série de números de cada vez e a repeti-la na mesma ordem. A resposta é considerada certa quando a criança é capaz de repetir corretamente toda a sequência de números que ouviu, seja na ordem direta ou inversa. Cada tarefa possui duas séries de números, apresentados como duas tentativas. A pontuação para cada item da OD e da OI é de 2 se a criança acertar as 2 tentativas, 1 se a criança acertar uma, 0 ponto se a criança errar ambas as tentativas. A pontuação para Dígitos OD é a soma dos pontos dos itens, totalizando 16 pontos brutos; para Dígitos OI, a soma dos pontos dos itens totaliza 14 pontos brutos. Dessa forma, o escore no subteste Dígitos é a soma dos pontos na OD e OI, considerando 30 pontos brutos. Os pontos brutos são transformados em ponderados em uma escala de 1 a 19, sendo esses a medida do subteste Dígitos.

O Subteste Aritmética. Avalia habilidades de cálculo, raciocínio matemático, capacidade de resolver as quatro operações básicas e a habilidade de resolução de problemas complexos. A criança deve resolver problemas matemáticos mentalmente, sem lápis e papel, mas pode usar os dedos para "escrever" na mesa. O subteste é formado por 24 itens apresentados em cartões, distribuídos em três conjuntos: 1) itens 1-5: problemas que se relacionam a gravuras são lidos para a criança; 2) itens 6-18: problemas desacompanhados por gravuras são lidos para a criança; 3) itens 19-24: a criança lê em voz alta os problemas. Nos itens 1-18 a criança possui 30 segundos para responder cada uma das questões; nos itens 19-24, 75 segundos. Cada item pode ser repetido uma vez, mediante a solicitação da criança ou se o examinador perceber que ela não compreendeu a questão. $\mathrm{O}$ tempo é marcado ao término da primeira leitura.

Com base no WISC-III, também foram calculados a VP e o índice de RD. Esses índices correspondem à soma dos pontos ponderados encontrados nos subtestes Código e Procurar Símbolos. A VP avalia os processos relacionados à atenção, memória e concentração para processar rapidamente a informação visual e o RD a resistência à distração, atenção e concentração, processamento sequencial. Lopes et al. (2012) apresentaram evidências de que crianças com TDAH possuem déficits nesses índices. Ademais, as recomendações da APA (2014), como já argumentado, sinalizam para esses "problemas cognitivos".

\section{Nomeação Seriada Rápida (NSR) de objetos,} letras, números e cores (Justi \& Roazzi, 2012). O conjunto de tarefas avalia precisão, automaticidade, sincronização e integração de processos de natureza per- 
ceptual (responsáveis pelo reconhecimento de padrões visuais), lexical (acesso e recuperação de informações semânticas, ortográficas e fonológicas) e motora (articulação da pronúncia dos estímulos), fundamentais para o bom desempenho em leitura. A tarefa se apresenta em quatro formas: cores, objetos, números e letras. Cada uma dessas tarefas é composta por 1 folha de papel A4 impressa e plastificada com cinco estímulos que se repetem 10 vezes de forma aleatória. Na forma cores, p. ex., apresenta-se à criança uma folha com as cores preto, branco, verde, vermelho e verde, que se repetem, aleatoriamente, em cinco fileiras horizontais. A mesma disposição é seguida para as demais folhas com os estímulos objetos, números e letras, separadamente. Os estímulos devem ser nomeados da esquerda para a direita, o mais rapidamente possível. Estudos nacionais têm investigado a relação da VN com a dificuldade de leitura e de escrita no TDAH, com achados que vão ao encontro dos resultados das pesquisas citadas na introdução (Bicalho \& Alves, 2009; Capellini, Ferreira, Salgado, \& Ciasca, 2007; Capellini \& Conrado, 2009; Cardoso-Martins, Vítor, \& Navas, 2010). Para a escolha da NSR, considerou-se essas pesquisas nacionais que avaliaram crianças com idade de 7 a 14 anos. Os pontos de corte para inclusão das crianças, respectivamente, mais novas e mais velhas, no GC foi de 50 segundos e 45 segundos em cores, $55 \mathrm{~s}$ e $50 \mathrm{~s}$ em objetos, $40 \mathrm{~s}$ e $35 \mathrm{~s}$ em números, e 40 s e 30 s em letras.

\section{Versão computadorizada do Stroop Palavra-Cor} (Duncan, 2006). É um dos testes mais conhecidos para avaliar a atenção seletiva e o controle inibitório, pois exige que informações já arquivadas na memória (no caso, palavras escritas) sejam inibidas para que a atenção seja focada em uma demanda do momento (a cor em que cada palavra é grafada). Tem sido sistematicamente explorado nas pesquisas sobre o TDAH (Assef, Capovilla, \& Capovilla, 2007; Mourik, Oosterlaan, \& Sergeant, 2005). Diante de uma palavra escrita, o participante é solicitado a dizer em voz alta a cor da tinta em que ela foi impressa, e não a ler a palavra. Por exemplo, diante da palavra verde, impressa na cor azul, a pessoa deve dizer azul e não a palavra verde. $O$ teste é composto por 26 slides: o slide 1 contém o nome do teste; e o 2, as suas instruções. Os 24 restantes, os itens do teste, aparecem automaticamente na tela do computador. O total de acertos é registrado, assim como o tipo de erro cometido. As crianças que não sabiam ler e não conheciam as cores básicas foram excluídas da amostra. O escore máximo é de 24 pontos (1 ponto por slide lido corretamente). O ponto de corte para inclusão no GC é de 19 pontos para as crianças mais novas e de 21 para as mais velhas.

Fluência Verbal Semântica (categoria animais) (FVS-Animais) e Fluência Verbal Fonológica (FAS) (Malloy-Diniz et al., 2007). As medidas de fluência verbal avaliam domínios específicos das funções executivas (FE): enquanto a tarefa FAS demanda a utilização de estratégias de busca, a de FVS-Animais pode identificar prejuízo na memória semântica. Na FVS-Animais o participante deve nomear o máximo de animais que conseguir em 1 minuto. Explica-se para criança que, no caso de nomear, p. ex., gato e gata, um deles é considerado erro, mas quando a denominação é cavalo e égua, ambas são corretas. Pontua-se individualmente o nome de cada subcategoria (p. ex., peixe ou pássaro). No entanto, se após "peixe" a criança citar tubarão, piranha etc., a subcategoria não é pontuada. $\mathrm{O}$ teste avalia linguagem (produção e fluência semântica), atenção sustentada, organização, uso de estratégia e perseveração. O ponto de corte para inclusão no GC é de 12 para as crianças mais novas e de 14 para as mais velhas. Na FAS, pede-se à criança que, durante 1 minuto, fale o número máximo de palavras que comece com as letras "F", "A" e "S", exceto nomes próprios e palavras derivadas. A tarefa avalia planejamento, organização, julgamento, atenção sustentada e linguagem. O ponto de corte para inclusão no GC é de 6 para as crianças mais novas e de 8 para as mais velhas.

Repetição de Pseudopalavras (Santos \& Bueno, 2003). O teste demanda a capacidade de memória fonológica para codificar e manter na memória uma 
sequência fonológica nova para subsequente produção dos movimentos articulatórios da fala. Essa tarefa avalia o estado da rota fonológica no processamento da fala, que inclui procedimentos de segmentação e síntese de fonemas. É composta por uma lista de 40 pseudopalavras, cada uma podendo conter de duas a cinco sílabas. As pseudopalavras são lidas para a criança e pede-se que ela repita o que ouviu logo em seguida. Foi usada uma folha como anteparo para impedir a leitura labial pela criança. $\mathrm{O}$ escore máximo estabelecido foi de 40 pontos. O ponto de corte inclusão no GC foi de 28 pontos para as crianças mais novas, e de 32 para as mais velhas.

Supressão de Fonemas (Lopes-Silva, Moura, JúlioCosta, Haase, \& Wood, 2014). Avalia a capacidade de representar e manipular conscientemente os fonemas nas palavras faladas, o que exige memória de trabalho e atenção (Cunha et al., 2013). O avaliador apresenta uma palavra (p. ex., perua), solicita que a criança subtraia mentalmente um som em particular (p. ex., u) e diga qual palavra se formou com sua retirada (pera). Foi usada uma folha como anteparo para impedir leitura labial. A tarefa é composta por 28 palavras e quatro itens adicionais de treino. Cada palavra respondida corretamente recebe um ponto, com escore total máximo de 28 . O ponto de corte inclusão no GC foi de 19 pontos para as crianças mais novas, e de 22 para as mais velhas.

\section{Procedimentos}

Coleta de dados. A coleta de dados envolveu uma média de cinco encontros com cada aluno. A escola disponibilizou horário e local adequados. Os procedimentos éticos em pesquisa com seres humanos foram atendidos, com a obtenção de termos de consentimento livre e esclarecido e assentimento das crianças e de seus pais, e termos de anuência institucional por parte das escolas. A pesquisa que originou o presente estudo foi aprovada pelo Comitê de Ética da Universidade Federal de Minas Gerais (parecer 686.858).

Análise dos dados. Os dados coletados foram analisados com base nos critérios indicados nos manuais dos instrumentos ou nas publicações relacionadas. A comparação de médias foi feita via ANOVA $(p<0,05)$, exceto para NSR_Letras, subteste Código e FAS_Letra A, para os quais foi utilizado o teste não paramétrico de Jonckheere para alternativas ordenadas (Ali et al., 2015). A análise de contrastes foi utilizada para identificar entre quais grupos se registram diferenças significativas. O conceito de significância prática fundamentou a interpretação do desempenho nas tarefas (Loureiro \& Gameiro, 2011). As análises foram conduzidas através do software SPSS (versão 19).

\section{Resultados}

Após a avaliação dos processos cognitivos afetados no TDAH e comorbidades, a prévia distribuição dos participantes em três grupos não se sustentou, uma vez que foram identificadas crianças com TDAH+Dislexia. Além disso, houve realocação de participantes entre os grupos originais. Desse modo, a amostra, após a reavaliação cujos resultados são apresentados a seguir, ficou distribuída do seguinte modo: Grupo 1, grupo controle (GC), sem TDAH nem sinais de dislexia, com idades entre 7 e 15 anos ( $(=45)$; Grupo 2, com TDAH, 7-13 anos $(\mathrm{n}=9)$; Grupo 3, com sinais de dislexia, 8-13 anos $(n=6)$; e Grupo 4, com TDAH e sinais de dislexia (doravante "grupo TDAH+Dislexia"), 8-12 anos ( $=11)$.

De início, pode-se notar que a quantidade de crianças anteriormente alocadas no GC aumentou de 31 para 45. Além disso, 20 alunos tiveram diagnóstico de TDAH confirmado pela avaliação realizada, dos quais 11 também apresentaram sinais de dislexia (TDAH+Dislexia). A avaliação de seis estudantes forneceu evidências de sinais de dislexia, mas não de TDAH. Desse modo, foi confirmada a hipótese de que a avaliação dos processos cognitivos empreendida neste trabalho seria capaz de detectar casos com sinais de dislexia, combinados ou não com o TDAH.

A Tabela 1 apresenta a comparação das médias dos quatro grupos para NSR (cores, objetos números e letras) e total de acertos no Stroop Palavra-Cor. O 


\section{Tabela 1}

Comparações de Grupos para Velocidade de Nomeação e Stroop Palavra-Cor

\begin{tabular}{|c|c|c|c|c|c|c|}
\hline \multirow{2}{*}{ Avaliação } & \multicolumn{4}{|c|}{ M (DP) } & \multirow{2}{*}{$\mathbf{p}$} & \multirow{2}{*}{ Contraste } \\
\hline & G1 & G2 & G3 & G4 & & \\
\hline NSR_Cores (s) & $\begin{array}{l}43,26 \\
(11,41)\end{array}$ & $\begin{array}{c}46,13 \\
(14,88)\end{array}$ & $\begin{array}{l}42,89 \\
(11,19)\end{array}$ & $\begin{array}{c}55,11 \\
(13,07)\end{array}$ & 0,040 & $1<4$ \\
\hline NSR_Objetos (s) & $\begin{array}{l}43,50 \\
(8,41)\end{array}$ & $\begin{array}{l}46,93 \\
(7,78)\end{array}$ & $\begin{array}{c}42,90 \\
(16,00)\end{array}$ & $\begin{array}{c}59,88 \\
(15,70)\end{array}$ & 0,001 & $1,2,3,<4$ \\
\hline NSR_Números (s) & $\begin{array}{l}29,36 \\
(7,21)\end{array}$ & $\begin{array}{l}27,73 \\
(7,38)\end{array}$ & $\begin{array}{c}35,46 \\
(12,31)\end{array}$ & $\begin{array}{l}36,15 \\
(8,26)\end{array}$ & 0,024 & $1<4$ \\
\hline NSR_Letras (s) & $\begin{array}{l}28,25 \\
(5,42)\end{array}$ & $\begin{array}{l}28,98 \\
(7,02)\end{array}$ & $\begin{array}{c}39,19 \\
(15,63)\end{array}$ & $\begin{array}{l}33,01 \\
(7,38)\end{array}$ & 0,057 & - \\
\hline $\begin{array}{l}\text { Stroop } \\
\text { (total acertos) }\end{array}$ & $\begin{array}{l}21,33 \\
(5,12)\end{array}$ & $\begin{array}{l}16,22 \\
(6,36)\end{array}$ & $\begin{array}{l}23,17 \\
(0,98)\end{array}$ & $\begin{array}{l}16,64 \\
(6,15)\end{array}$ & 0,005 & $1>4$ \\
\hline
\end{tabular}

Nota: $\mathrm{M}$ = média; DP = desvio-padrão; NSR = nomeação seriada rápida; s = segundos; G1 = sem transtorno; G2 = TDAH; G3 = dislexia; G4 = TDAH+Dislexia.

contraste aponta a diferença significativa nas relações entre os grupos.

Conforme a Tabela 1, na tarefa NSR_Cores a diferença foi significativa entre o Grupo 1 e o Grupo 4, com os primeiros sendo mais velozes nessa tarefa. Em NSR_Objetos, os grupos 1, 2 e 3 foram mais rápidos do que o Grupo 4. Na NSR_Letras, as diferenças entre os grupos não foram significativas. Na tarefa Stroop, o contraste do Grupo 4 com o Grupo 1 foi significativo.

A Tabela 2 mostra a comparação das médias dos quatro grupos para os subtestes e índices do WISCIII utilizados no estudo: Código, Dígitos, Procurar Símbolos, Aritmética, RD e VP. Os dados têm origem nos pontos ponderados.

\section{Tabela 2}

Comparações de Grupos para os Subtestes e Índices do WISC-III

\begin{tabular}{|c|c|c|c|c|c|c|}
\hline \multirow{2}{*}{ Avaliação } & \multicolumn{4}{|c|}{ M (DP) } & \multirow{2}{*}{$\mathbf{p}$} & \multirow{2}{*}{ Contraste } \\
\hline & G1 & G2 & G3 & G4 & & \\
\hline Código & $\begin{array}{c}11,67 \\
(2,82)\end{array}$ & $\begin{array}{c}9,67 \\
(2,69)\end{array}$ & $\begin{array}{c}11,33 \\
(5,54)\end{array}$ & $\begin{array}{c}7,36 \\
(2,34)\end{array}$ & 0,001 & $1>4$ \\
\hline Procurar símbolos & $\begin{array}{l}12,27 \\
(2,56)\end{array}$ & $\begin{array}{l}11,67 \\
(2,78)\end{array}$ & $\begin{array}{c}11,17 \\
(2,99)\end{array}$ & $\begin{array}{c}10,27 \\
(3,88)\end{array}$ & 0,208 & - \\
\hline Dígitos & $\begin{array}{l}11,24 \\
(3,74)\end{array}$ & $\begin{array}{c}9,11 \\
(1,90)\end{array}$ & $\begin{array}{c}7,50 \\
(1,87)\end{array}$ & $\begin{array}{c}8,27 \\
(2,65)\end{array}$ & 0,001 & $1>4$ \\
\hline OD_Dígitos & $\begin{array}{c}5,44 \\
(1,45)\end{array}$ & $\begin{array}{c}4,67 \\
(1,00)\end{array}$ & $\begin{array}{c}4,33 \\
(0,82)\end{array}$ & $\begin{array}{c}4,55 \\
(0,82)\end{array}$ & 0,047 & $1>4$ \\
\hline OI_Dígitos & $\begin{array}{c}3,67 \\
(1,11)\end{array}$ & $\begin{array}{c}2,67 \\
(0,71)\end{array}$ & $\begin{array}{c}2,50 \\
(0,55)\end{array}$ & $\begin{array}{c}2,73 \\
(0,65)\end{array}$ & 0,001 & $1>2,3,4$ \\
\hline Aritmética & $\begin{array}{c}11,73 \\
(3,14)\end{array}$ & $\begin{array}{l}10,67 \\
(3,00)\end{array}$ & $\begin{array}{c}8,17 \\
(4,22)\end{array}$ & $\begin{array}{c}8,27 \\
(2,41)\end{array}$ & 0,003 & $1>4$ \\
\hline
\end{tabular}




\begin{tabular}{|l|l|l|l|l|l|l|}
\hline RD & $\begin{array}{l}23,18 \\
(5,75)\end{array}$ & $\begin{array}{c}20,56 \\
(5,13)\end{array}$ & $\begin{array}{c}15,67 \\
(5,01)\end{array}$ & $\begin{array}{c}16,45 \\
(3,98)\end{array}$ & 0,001 & $1>3,4$ \\
\hline VP & $\begin{array}{l}23,87 \\
(4,93)\end{array}$ & $\begin{array}{c}20,56 \\
(4,22)\end{array}$ & $\begin{array}{c}22,33 \\
(8,31)\end{array}$ & $\begin{array}{c}17,64 \\
(4,84)\end{array}$ & 0,005 & $1>4$ \\
\hline
\end{tabular}

Nota. Nessa análise foram considerados os pontos ponderados. $\mathrm{M}=$ média; $\mathrm{DP}=$ desvio-padrão; $\mathrm{OD}=$ ordem direta; $\mathrm{OI}=$ ordem inversa; RD = resistência à distração; VP = velocidade de processamento; G1 = sem transtorno; G2 = TDAH; G3 = dislexia; G4 = TDAH+Dislexia.

Como mostra a Tabela 2, os grupos não diferiram significativamente em Procurar Símbolos. Nas medidas Código, Dígitos, Dígitos OD, Aritmética e VP, o Grupo 1 apresentou escore significativamente maior que o Grupo 4.

$\mathrm{O}$ índice $\mathrm{RD}$ se mostrou uma evidência importante sobre os grupos 3 e 4 . As médias desses grupos, segundo a Tabela 2, foram abaixo do previsto pelo WISC-III, bem como significativamente inferiores ao Grupo 1.

Na Tabela 3 constam os resultados para as comparações de médias do total de acertos para as avaliações de fluência verbal semântica e fonológica, Repetição de Pseudopalavras e Supressão de Fonemas. Não foram encontradas diferenças significativas entre os grupos na avaliação da FVS-Animais nem da FAS_Letra S.
A Tabela 3 mostra que, na maioria das avaliações, o Grupo 1 contrastou-se do Grupo 4. Mais especificamente, os escores de FAS_Letra F, FAS_Letra A, Supressão de Fonemas e Repetição de Pseudopalavras foram significativamente maiores no Grupo 1 quando comparado ao Grupo 4.

Como esperado, na maioria das tarefas o Grupo 1 diferiu significativamente do Grupo 4. Ademais, o Grupo 1 demonstrou desempenho superior em relação aos demais grupos no subteste Dígitos OI, que mede MT e habilidades relacionadas ao controle da atenção e de seleção de estratégias. Outro destaque do Grupo 1 foi o menor DP na maioria das tarefas, mostrando, como esperado, se tratar de um grupo mais homogêneo, apesar da amplitude etária da amostra (7-15 anos).

\section{Tabela 3}

Comparações de Grupos para Fluência Verbal, Repetição de Pseudopalavras e Supressão de Fonemas

\begin{tabular}{|c|c|c|c|c|c|c|}
\hline \multirow{2}{*}{ Avaliação } & \multicolumn{4}{|c|}{ M (DP) } & \multirow{2}{*}{$\mathbf{p}$} & \multirow{2}{*}{ Contraste } \\
\hline & G1 & G2 & G3 & G4 & & \\
\hline FVS-Animais & $\begin{array}{l}13,20 \\
(3,27)\end{array}$ & $\begin{array}{c}13,11 \\
(3,22)\end{array}$ & $\begin{array}{c}11,17 \\
(3,87)\end{array}$ & $\begin{array}{l}11,55 \\
(2,16)\end{array}$ & 0,260 & - \\
\hline FAS_Letra F & $\begin{array}{c}7,02 \\
(3,07)\end{array}$ & $\begin{array}{c}6,44 \\
(3,54)\end{array}$ & $\begin{array}{c}4,33 \\
(2,66)\end{array}$ & $\begin{array}{c}4,45 \\
(2,34)\end{array}$ & 0,032 & $1>4$ \\
\hline FAS_Letra A & $\begin{array}{c}6,47 \\
(2,63)\end{array}$ & $\begin{array}{c}5,78 \\
(2,39)\end{array}$ & $\begin{array}{c}4,50 \\
(1,05)\end{array}$ & $\begin{array}{c}4,55 \\
(1,51)\end{array}$ & 0,006 & $1>4$ \\
\hline FAS_Letra S & $\begin{array}{c}6,31 \\
(2,12)\end{array}$ & $\begin{array}{c}5,33 \\
(2,18)\end{array}$ & $\begin{array}{c}4,50 \\
(2,59)\end{array}$ & $\begin{array}{c}4,82 \\
(1,83)\end{array}$ & 0,064 & - \\
\hline Repetição de Pseudopalavras & $\begin{array}{l}37,49 \\
(2,30)\end{array}$ & $\begin{array}{l}35,89 \\
(2,47)\end{array}$ & $\begin{array}{l}35,50 \\
(1,87)\end{array}$ & $\begin{array}{l}33,64 \\
(3,56)\end{array}$ & 0,001 & $1>4$ \\
\hline Supressão de Fonemas & $\begin{array}{l}22,40 \\
(5,41)\end{array}$ & $\begin{array}{l}17,78 \\
(7,31)\end{array}$ & $\begin{array}{l}16,50 \\
(6,83)\end{array}$ & $\begin{array}{l}12,91 \\
(7,76)\end{array}$ & 0,001 & $1>4$ \\
\hline
\end{tabular}

Nota . Nessa análise foi considerado o total de acertos. $\mathrm{M}=$ média; DP = desvio-padrão; FVS-Animais = Fluência Verbal Semântica (categoria animais) FAS = Fluência verbal fonológica; G1 = sem transtorno; G2 = TDAH; G3 = dislexia; G4 = TDAH+Dislexia. 
Com relação ao Grupo 2 (com TDAH), o que o distinguiu dos grupos 1 e 3 foi o desempenho inferior em NSR_Cores e NSR_Objetos, no Stroop e no Código. Ademais, o Grupo 2 foi inferior do que ao $1 \mathrm{em}$ Dígitos e em Supressão de Fonemas. Esses resultados indicam que o critério para a confirmação do status "com TDAH" do Grupo 2 foi atingido.

O Grupo 3 (com sinais de dislexia) apresentou desempenho inferior em seis escores, na comparação com os grupos 1 e 2. Foram eles: NSR_Números, NSR Letras, Supressão de Fonemas, Dígitos, Aritmética e RD.

Conforme antecipado, o Grupo 4 se distinguiu dos demais pelo desempenho inferior em todos os instrumentos que avaliam funções cognitivas usualmente deficitárias no TDAH e na dislexia. Em NSR_Objetos, a média do Grupo 4 foi a maior (59,88 s); em NSR_Números, o Grupo 4 novamente apresentou maior lentidão. Já em VP, os grupos 2 e 4 mostraram desempenhos inferiores em relação aos demais grupos. Outra característica do Grupo 4 foi a menor média em relação ao Grupo 3 em Supressão de Fonemas.

As tarefas de FVS-Animais e FAS constituíram maior desafio tanto para o Grupo 3 como para o Grupo 4. Nessas tarefas, as médias do Grupo 2, mesmo menores, se aproximaram das médias do Grupo 1.

Os resultados mostram que tanto o Grupo 2 como o Grupo 4 apresentaram desempenho inferior nas tarefas e testes aplicados na comparação com o Grupo 1. Assim, foi também confirmada a hipótese de que os participantes com TDAH (presentes nos grupos 2 e 4 ) apresentariam desempenho inferior em termos de MT, VP, NSR, controle inibitório e atenção, na comparação com o Grupo 1. Mais que isso, observou-se que os participantes com TDAH+Dislexia apresentaram prejuízo destacado nos processos cognitivos avaliados.

\section{Discussão}

Os resultados deste trabalho confirmam a contribuição da avaliação neuropsicológica para aumentar a precisão do diagnóstico do TDAH (Larroca \& Domingos,
2012; Lopes et al., 2012). Os instrumentos utilizados se mostraram eficazes para a investigação de casos com indicadores de dislexia, inclusive na combinação com o TDAH, principalmente dada a carência de testes psicométricos no Brasil para o rastreio do TDAH e da dislexia.

De todo modo, foi possível identificar os instrumentos que parecem ser confiáveis para a realização do diagnóstico neuropsicológico do TDAH, indicadores de dislexia e da combinação de TDAH e dislexia. Para o TDAH, a tarefa Stroop é um dos principais marcadores. Outros instrumentos importantes são as de NSR_Cores e NSR_Objetos, os subtestes do WISC utilizados neste estudo, assim como os índices de VP e de RD.

Já para os indicadores de dislexia, além das tarefas clássicas de NSR_Letras e NSR_Números e processamento fonológico, os subtestes Dígitos, Aritmética e o índice RD se mostraram importantes medidas, com destaque para o Dígitos. As crianças com sinais de dislexia apresentaram desempenho inferior nos subtestes Dígitos e Aritmética, e em RD.

Os resultados da tarefa NSR_Letras colaboram para ilustrar a relevância do conceito de significância prática. Dado que a tarefa de NSR é preditora para o desenvolvimento da leitura e da escrita (Norton \& Wolf, 2012) e que o processamento da linguagem e da cognição, de forma geral, ocorre em milésimos de segundo (Lúcio, Pinheiro, \& Nascimento, 2009), eram esperadas diferenças educacionais e clínicas entre os grupos no que se refere à habilidade medida por essa tarefa.

Com a identificação dos processos cognitivos preservados e deficitários rastreados pelos instrumentos utilizados no presente estudo, é possível fazer uma descrição de um conjunto de condições cognitivas para cada um dos grupos que incluiu estudantes em condição de risco neurodesenvolvimental: grupo com TDAH, grupo com sinais de dislexia, e grupo com TDAH e sinais de dislexia. Especialmente, com relação a esse último, Rucklidge e Tannock (2002) chamam a atenção para a forma como os déficits cognitivos (NSR_Objetos, 
NSR_Números e VP) se imbricam nessa condição, o que poderia lhes conferir o perfil de um subgrupo específico.

\section{O Perfil Cognitivo dos Escolares com TDAH}

O desempenho deficitário dos estudantes com TDAH no Stroop está de acordo com McGrath et al. (2011) sobre o controle inibitório (medido por essa tarefa) ser preditor específico desse transtorno. Se esse déficit é causado por uma disfunção das FE, essas funções devem também estar prejudicadas na maior parte dos alunos com TDAH, inclusive com sinais de dislexia. De fato, o subteste Dígitos OI, relacionado ao executivo central, diferenciou o Grupo 1 (crianças sem TDAH nem sinais de dislexia) dos demais grupos. Por esse subteste demandar a capacidade de distribuição da atenção, de selecionar estratégias e de coordenar diferentes processos, é possível que as deficiências nesses processos, pelo menos nos casos com TDAH, estejam subjacentes ou relacionadas ao reduzido controle inibitório.

Prejuízos nas habilidades medidas pelo subteste Código e pelo índice VP foram também importantes na caracterização do perfil cognitivo com TDAH. O Código avalia atenção seletiva, organização perceptiva, memória episódica, desempenho psicomotor e acurácia na coordenação mão-olho, demandando atenção sustentada, velocidade de resposta e coordenação visomotora. Já o índice VP exige atenção, memória e concentração para processar rapidamente a informação visual. Em conjunto, deficiências nessas habilidades levam o estudante a empregar mais tempo em tarefas cotidianas, incluindo a leitura. A propósito, Albuquerque, Maia, França, Mattos e Pastura (2012) demonstraram que crianças com TDAH, embora lessem palavras e frases, levavam mais tempo para executar essas atividades do que crianças sem o transtorno.

Os escolares com TDAH apresentaram também deficiências na MT, expressas por desempenho ineficaz no subteste Dígitos e no índice de RD, confirmando os achados de Martinussen e Tannock (2006) e de McGrath et al. (2011). Lopes et al. (2012) também encontraram desempenho inferior da criança com TDAH no WISC-III (distintos subtestes e nos índices $R D$ e $V P$ ).

Por fim, o grupo com TDAH apresentou desempenho inferior nas quatro tarefas de NSR em relação ao grupo de crianças sem TDAH nem sinais de dislexia. O grupo com sinais de dislexia foi inferior ao do com TDAH apenas na NSR_Letras. A NSR é sensível à habilidade de integrar processos cognitivos subjacentes à leitura, à automação de processos cognitivos, discriminação visual e recuperação de padrões fonológicos. Além da disfunção nesses processos, o grupo com TDAH apresentou desempenho inferior em Supressão de Fonemas, evidenciando dificuldades em manipular os sons da fala. Esse e outros achados da presente pesquisa fortalecem a hipótese de déficit de linguagem no escolar com TDAH (Prando et al., 2013; Rucklidge \& Tannock, 2002). Por fim, a detecção de déficits em vários processos cognitivos no TDAH apoia o modelo de múltiplos déficits (McGrath et al., 2011; Pennington, 2006).

\section{TDAH versus Dislexia}

As medidas que distinguiram os escolares com sinais de dislexia (Grupo 3) do grupo com TDAH (grupo 2) foram: NSR_Letras, NSR_Números, Dígitos, Aritmética, RD e Supressão de Fonemas. Em termos de VP, o grupo com sinais de dislexia, embora tivessem apresentado um desempenho melhor do que o grupo com TDAH, foram piores na comparação com o sem transtorno. Esse resultado está de acordo com McGrath et al. (2011) que afirmam que os preditores específicos para a dislexia são déficits na NSR e na consciência fonêmica. Além disso, um déficit na VP é preditor para TDAH e para dislexia, o que está de acordo com Nikolas e Nigg (2013) e Pennington (2006). Para estes autores, o padrão de déficits específicos a cada condição oferece evidência para o modelo de múltiplos déficits cognitivos para o TDAH, para a dislexia e para a comorbidade entre essas condições.

Os resultados do grupo com sinais de dislexia, em comparação com as crianças TDAH, também foram piores em Dígitos, Aritmética, RD e FVS-Animais, indi- 
cando problemas adicionais. Os escores na NSR_Números e no subteste Aritmética oferecem evidências de que o disléxico possa apresentar dificuldade em matemática (Cruz-Rodrigues, Barbosa, Toledo-Piza, Miranda, \& Bueno, 2014) e sugerem comorbidade com discalculia. Esse achado vai ao encontro da revisão apresentada por Silva, Moura, Wood e Haase (2015). Nela evidenciou-se que investigações, mais detalhadas, realizadas nas últimas décadas identificam o pior desempenho matemático de disléxicos. O raciocínio matemático envolve o código verbal, a recuperação de um cálculo matemático depende da qualidade da representação fonológica desse cálculo. Assim, um déficit na consciência fonológica pode levar a dificuldades com o raciocínio matemático.

A habilidade quanto à $\mathrm{MT}$, testada pelo subteste Dígitos OI, foi crucial para distinguir o grupo sem transtorno dos demais. Crianças com TDAH e crianças disléxicas apresentam baixo desempenho em medidas de MT (Martinussen \& Tannock, 2006). Contudo, Cohen et al. (2000) destacam que a MT na dislexia é pior do que no TDAH. Os achados do presente estudo confirmam Cohen et al. (2000), mas não para Dígitos OI, uma vez que as médias do grupo com TDAH e do grupo com sinais de dislexia foram muito próximas.

Foi considerável a dissociação entre os pares NSR_Cores e NSR_Objetos com os NSR_Letras e NSR_Números, o que está de acordo com Tannock et al. (2000) e com Norton \& Wolf (2012). Esses últimos reportam uma dissociação entre o par NSR_Cores e NSR_Objetos com NSR_Letras apenas. Esses resultados apontam para disfunções nos processos gerais medidos na NSR, os quais são responsáveis pela precisão, automaticidade, sincronização e integração de processos de natureza perceptual, lexical e motora utilizados no reconhecimento de padrões visuais, e no acesso e recuperação de informações semânticas, ortográficas e fonológicas que possibilitam a articulação da pronúncia dos estímulos. Para Norton e Wolf, o construto VN mede uma habilidade cognitiva única, cujos déficits não podem ser associados apenas à dislexia e ao processa- mento fonológico (Tannock et al., 2000). No entanto, a dissociação encontrada nessas tarefas, e referida na literatura, carece de explicação definitiva.

Destaca-se também que o grupo com sinais de dislexia apresentou melhor desempenho no Stroop do que os indivíduos do GC. Esse achado, imprevisto no estudo, sugere que essa tarefa colabora para diferenciar indivíduos com sinais de dislexia daqueles com TDAH.

\section{O Perfil Cognitivo do Escolar com TDAH e Dislexia}

O grupo TDAH+Dislexia mostrou comprometimentos nas habilidades que foram marcadores tanto para o grupo só com TDAH puro, quanto para o grupo com sinais de dislexia. Esse achado oferece evidência para a realidade psicológica do perfil comórbido. O baixo desempenho dos grupos de TDAH (Grupos 2 e 4) em Supressão de Fonemas, uma das tarefas clássicas para o diagnóstico de dislexia, foi inesperado. Uma vez que o sucesso nessa tarefa demanda a utilização de MT e de atenção, o resultado encontrado oferece suporte para as pesquisas que tributam a disfunção da linguagem no TDAH a déficit da MT ou mesmo do processamento atentivo (Katz et al., 2011; Miranda et al., 2012). Mostra também que é necessário ampliar a investigação da linguagem no TDAH de forma, por um lado, a distinguir as crianças com essa condição daquelas com distúrbios gerais de linguagem e com distúrbios específicos (ou dislexia) (Miller et al., 2013); por outro lado, identificar a comorbidade TDAH+Dislexia.

Assim, os resultados para o grupo TDAH+Dislexia reforçam a posição de Rucklidge e Tannock (2002) acerca da necessidade de se ver esse grupo como possuindo um perfil cognitivo específico. No presente estudo essa condição comórbida apresentou pior desempenho nas tarefas NSR_Cores, NSR_Objetos, NSR_Números, VP e Supressão de Fonemas. Ademais, o fracasso nessa última tarefa confirma que os déficits cognitivos das crianças com TDAH+Dislexia sobrepõem-se de forma a desfavorecê-las, uma vez que apresentaram desempenho médio inferior ao do grupo de crianças disléxicas. 


\section{Considerações finais}

Todos os estudos que discutem a prevalência do TDAH no Brasil referem a dificuldade da realização do diagnóstico dessa condição e da falta de acordo sobre a sua prevalência. Considerando-se que "a dificuldade de se fazer um diagnóstico confiável de TDAH tem prejudicado a interpretação das estimativas de prevalência desse transtorno" (Moraes, 2010, p. 37) e gerado consequências negativas nos níveis pessoais, familiares e sociais dos indivíduos com o TDAH, a presente pesquisa, focalizando nas questões relacionadas ao diagnóstico do TDAH, abordou, principalmente, a identificação dos instrumentos que rastreiam os processos cognitivos preservados e deficitários no TDAH e em comorbidade com a dislexia, e uma descrição do perfil cognitivo dos indivíduos com TDAH com base no desempenho nas medidas cognitivas.

O método empregado consistiu na identificação de um conjunto de instrumentos para reavaliar as crianças julgadas pelas professoras como em risco de apresentar TDAH e aquelas com diagnóstico prévio (médico ou psicológico) de TDAH e de distingui-las de crianças disléxicas de forma a descrever os perfis de escolares gerados. Confirmada a primeira hipótese do estudo, em comparação com os controles e disléxicos, os escolares com TDAH mostraram reduzido controle inibitório (medido pela tarefa Stroop), assim como prejuízos nas habilidades avaliadas pelo índice VP, sendo as principais delas a atenção seletiva e sustentada, a MT e a concentração para processar rapidamente a informação. Com relação à memória mostram especial deficiência na MT (medida pelo subteste Dígitos OI), seguido de desempenho insatisfatório nas tarefas de NSR (Cores e Números) e, contrário às expectativas, também na tarefa de Supressão de Fonemas. O desempenho inferior nessa última tarefa permite, dentre outras distinções importantes (TDAH vs. distúrbios gerais da linguagem e TDAH vs. dislexia), a identificação da comorbidade TDAH+Dislexia, o que nos leva à confirmação da segunda hipótese do estudo.
Especificamente, por meio da avaliação conduzida, além das crianças com TDAH, foi possível identificar os alunos com sinais de dislexia e os casos de comorbidade. Aqueles com sinais de dislexia se sobressaíram por prejuízos em NSR_Letras, NSR_Números, Dígitos (em especial OI), Aritmética, RD e Supressão de Fonemas, sendo que os preditores específicos para a dislexia são déficits na NSR e na consciência fonêmica. Observa-se que semelhantemente às crianças com TDAH, as com sinais de dislexia apresentam baixo desempenho em medidas de MT. Já a condição comórbida TDAH+Dislexia, se caracteriza por apresentar marcadores tanto para o grupo com TDAH puro (comprometimento na tarefa Stroop) quanto para o grupo com sinais de dislexia (comprometimento em NSR_Cores, NSR_Objetos, NSR_Números, VP e Supressão de Fonemas), revelando um perfil cognitivo específico.

Sobre as limitações do presente estudo, embora a amostra geral utilizada esteja de acordo com o que se encontra na literatura, ao separá-la em diferentes condições, os grupos clínicos ficaram com um número limitado de participantes. Isso impossibilita a generalização dos achados do estudo, mesmo estando eles de acordo com a literatura.

Os achados evidenciaram a necessidade de aperfeiçoamento da rede pública de ensino e de saúde para o atendimento da criança com TDAH e comorbidades. Isso deve ser feito não só por meio do investimento na qualificação dos profissionais envolvidos, mas, especialmente, garantindo o funcionamento do sistema público como uma rede de serviços. Somente na medida em que os governos aproximarem os seus discursos e as ações dos achados científicos que investigam os transtornos neurodesenvolvimentais, eles cumprirão, de fato, com o seu papel de gestores do bem-estar da criança e do adolescente. Condições devem ser criadas para que os profissionais que compõem essa rede pública de serviços (especialmente médicos e psicólogos) atuem intersetorialmente. Além disso, entende-se necessário promover a entrada de profissionais no serviço público com maior 
conhecimento sobre o TDAH, ancorados nas pesquisas científicas sobre esse transtorno. Somente esse conjunto de mudanças no sistema público de educação e de saúde poderá ajudar a criança com TDAH a escapar da exclusão escolar e social, diminuindo o seu sofrimento e o da sua família. Mesmo que esta pesquisa não tenha abordado diretamente o sofrimento psíquico dessa criança, sabe-se que o sentimento de fracasso e impotência são presenças constantes na vida da pessoa que apresenta esse transtorno.

Por fim, os diferentes déficits cognitivos que a criança com TDAH+Dislexia apresenta justificam os achados sobre o fracasso escolar ao qual ela está sujeita. A escola não tem conseguido reconhecer os sinais de dislexia nessa criança, nem utilizado práticas pedagógicas que a ajudem a superar as suas limitações. De fato, há muito tempo a ciência da leitura evidencia que a estimulação precoce da criança disléxica evita o surgimento dos sintomas mais graves desse transtorno. A educação brasileira precisa acompanhar mais de perto o avanço científico na ciência da leitura.

\section{Referências}

Abujadi, C., \& Moraes, C. (2010). Instrumentos estruturados de avaliação do Transtorno de Déficit de Atenção e Hiperatividade. In S. Ciasca, S. Rodrigues, \& C. Salgado (Eds.), TDAH: Transtorno de Déficit de Atenção e Hiperatividade (pp. 141-149). Rio de Janeiro: Revinter. https://doi.org/10.11606/d.47.2009.tde-21032009-095322

Albuquerque, G., Maia, M., França, A., Mattos, P., \& Pastura, G. (2012). Processamento da linguagem no Transtorno do Déficit de Atenção e Hiperatividade (TDAH). DELTA (online), 28(2), 245-280. http://dx.doi. org/10.1590/S0102-44502012000200003

Ali, A., Rasheed, A., Siddiqui, A., Naseer, M., Wasim, S., \& Akhtar, W. (2015). Non-parametric test for ordered medians: The JonckheereTerpstra test. International Journal of Statistics in Medical Research, 4, 203-207. http://dx.doi.org/10.6000/1929-6029.2015.04.02.6

APA. AmericanPsychiatric Association (2014). DSM5: Manual Diagnóstico e Estatístico de Transtornos Mentais ( $5^{\mathrm{a}}$ ed.). Porto Alegre: Artmed.
Assef, E., Capovilla, A., \& Capovilla, F. (2007). Computerized Stroop Test to assess selective attention in children with Attention Deficit Hyperactivity Disorder. The Spanish journal of psychology,10(1), 33-40. https:// doi.org/10.1017/S1138741600006296

Associação Brasileira de Empresas de Pesquisa (2013). Critério de classificação econômica Brasil. Recuperado em agosto, 2017, de http://www.abep.org

Bicalho, L., \& Alves, L. (2009). A nomeação seriada rápida em escolares com e sem queixas de problemas de aprendizagem em escola pública e particular. Revista CEFAC, 12(4), 608-616. https://dx.doi.org/10.1590/S151618462010005000018

Capellini, S., \& Conrado, T. (2009). Desempenho de escolares com e sem dificuldades de aprendizagem de ensino particular em habilidade fonológica, nomeação rápida, leitura e escrita. Revista CEFAC, 11(2), 183-193. http://dx.doi.org/10.1590/S1516-18462009005000002

Capellini, S., Ferreira, T., Salgado, C., \& Ciasca, S. (2007). Desempenho de escolares bons leitores, com dislexia e com Transtorno de Déficit de Atenção e Hiperatividade em nomeação automática rápida. Revista da Sociedade Brasileira de Fonoaudiologia, 12(2),114-119. https:// dx.doi.org/10.1590/S1516-80342007000200008

Cardoso-Martins, C., Vítor, R., \& Navas, A. (2010). Distúrbios do desenvolvimento da fala e habilidade inicial de leitura e escrita em português: O papel da velocidade de nomeação. In M. Rodrigues, \& T. Sperb (Eds.), Contextos de desenvolvimento da linguagem (pp. 159-180). São Paulo: Vetor. https://doi.org/10.17771/ pucrio.acad. 35822

Cohen, N., Vallance, D., Barwick, M., Im, N., Menna, R., Horodezky, N., \& Isaacson, L. (2000).The interface between ADHD and language impairment: An examination of language, achievement, and cognitive processing. Journal Child Psychology and Psychiatry, 41(3), 353-362. http://dx.doi.org/10.1111/1469-7610.00619

Cruz-Rodrigues, C., Barbosa, T., Toledo-Piza, C., Miranda, M., \& Bueno, O. (2014). Neuropsychological characteristics of dyslexic children. Psicologia: Reflexão e Crítica, 27(3), 539-546. https://dx.doi.org/10.1590/1678$\underline{7153.201427315}$

Cunha, V., Silva, C., Lourencetti, M., Padula, A., \& Capellini, S. (2013). Desempenho de escolares com transtorno de déficit de atenção e hiperativida- 
de em tarefas metalinguísticas e de leitura. Revista CEFAC, 15(1), 40-50. https://dx.doi.org/10.1590/S1516$\underline{18462012005000003}$

Duncan, M. (2006). Obtenção de dados normativos para desempenho no teste de Stroop num grupo de estudantes do ensino fundamental em Niterói. Jornal Brasileiro de Psiquiatria, 55(1), 42-48. https://doi.org/10.1590/ s0047-20852006000100006

Figueiredo, V., \& Nascimento, E. (2007). Desempenhos nas duas tarefas do subteste dígitos do WISC-III e do WAIS-III. Psicologia: Teoria e Pesquisa, 23(3), 313-318. https://dx.doi.org/10.1590/S0102-37722007000300010

Justi, C., \& Roazzi, A. (2012). A contribuição de variáveis cognitivas para a leitura e a escrita no português brasileiro. Psicologia: Reflexão e Crítica, 25(3), 605-614. http://dx.doi.org/10.1590/S0102-79722012000300021

Katz, L., Brown, F., Roth, R., \& Beers, S. (2011). Processing speed and working memory performance in those with both ADHD and a reading disorder compared with those with ADHD alone. Archives of Clinical Neuropsychology, 26, 425-433. https://10.1093/ arclin/acr026

Larroca, L., \& Domingos, N. (2012). TDAH: Investigação dos critérios para diagnóstico do subtipo predominantemente desatento. Psicologia Escolar e Educacional, 16(1), 113-123. http://dx.doi.org/10.1590/ $\underline{\text { S1413-85572012000100012 }}$

Lopes, R., Farina, M., Wendt, G., Esteves, C., \& Argimon, I. (2012). Sensibilidade do WISC-III na identificação do Transtorno de Déficit de Atenção/Hiperatividade (TDAH). Cuadernos de Neuropsicología, 6(1), 128-140 https://doi.org/10.11606/d.47.2009.tde-21032009-095322

Lopes-Silva, J., Moura, R., Júlio-Costa, A., Haase, V., $\&$ Wood, G. (2014). Phonemic awareness as a pathway to number transcoding. Frontiers in Psychology, 5, 13. http://doi.org/10.3389/fpsyg.2014.00013

Loureiro, L., \& Gameiro, M. (2011). Interpretação crítica dos resultados estatísticos: Para lá da significância estatística. Revista de Enfermagem Referência, III(3), 151-162. http://dx.doi.org/10.12707/RIII1009

Lúcio, P., Pinheiro, A., \& Nascimento, E. (2009). A influência de fatores sociais, individuais e linguísticos no desempenho de crianças na leitura em voz alta de palavras isoladas. Psicologia: Reflexão e Crítica, 23(3), 496-505. http://dx.doi.org/10.1590/S0102-79722010000300010

Malloy-Diniz, L., Bentes, R., Figueiredo, P., BrandãoBretas, D., Costa-Abrantes, S., Parizzi, A., ... Salgado, J. (2007). Normalización de una batería de tests para evaluar las habilidades de comprensión del lenguaje, fluidez verbal y denominación en niños brasileños de 7 a 10 años: Resultados preliminares. Revista de Neurología, 44(5), 275-280. https://doi.org/10.33588/ $\underline{\text { rn.4405.2006089 }}$

Martinussen, R., \& Tannock, R. (2006).Working memory impairments in children with attention-deficit hyperactivity disorder with and without comorbid language learning disorders. Journal of Clinical and Experimental Neuropsychology, 28(7), 1073-1094. http:// dx.doi.org/10.1080/13803390500205700

Mattos, P., Serra-Pinheiro, M., Rohde, L., \& Pinto, D. (2006). Apresentação de uma versão em português para uso no Brasil do instrumento MTA-SNAP-IV de avaliação de sintomas de Transtorno de Déficit de Atenção/ Hiperatividade e sintomas de Transtorno Desafiador e de Oposição. Revista de Psiquiatria do Rio Grande do Sul, 28(3), 290-297. http://dx.doi.org/10.1590/S0101$\underline{81082006000300008}$

McGrath, L., Pennington, B., Shanahan, M., SanterreLemmon, L., Barnard, H., Willcutt, E., ... Olson, R. (2011). A multiple deficit model of reading disability and Attention-Deficit/Hyperactivity Disorder: Searching for shared cognitive deficits. Journal of Child Psychology and Psychiatry, 52(5), 547-557. http://dx.doi.org/10.1111/ j.1469-7610.2010.02346.x

Miller, A., Keenan, J., Betjemann, R., Willcutt, E., Pennington, B., \& Olson R. (2013). Reading comprehension in children with ADHD: Cognitive underpinnings of the centrality deficit. Journal of Abnormal Child Psychology, 41, 473-483. https://doi.org/10.1007/ $\underline{\text { s10802-012-9686-8 }}$

Miranda, M., Barbosa, T., Muszkat, M., Rodrigues C., Sinnes, E., Coelho, L., ... Bueno, O. (2012). Performance patterns in Conners' CPT among children with Attention Deficit Hyperactivity Disorder and dyslexia. Arquivos de Neuropsiquiatria, 70(2), 91-96. http://dx.doi.org/10.1590/ S0004-282X2012000200004

Moraes, C. (2010). Transtorno de Déficit de Atenção e Hiperatividade: Quadro clínico e comorbidades psi- 
quiátricas. In S. Ciasca, S. Rodrigues, \& C. Salgado (Eds.), TDAH: Transtorno de Déficit de Atenção e Hiperatividade (pp. 37-54). Rio de Janeiro: Revinter. https://doi.org/10.11606/d.47.2009.tde-21032009-095322

Mourik, R., Oosterlaan, J., \& Sergeant, J. (2005). The Stroop revisited: A meta-analysis of interference control in AD/HD. Journal of Child Psychology and Psychiatry, 46(2), 150-165. https://doi.org/10.1111/j. 1469-7610.2004.00345.x

Nikolas, M., \& Nigg, J. (2013). Neuropsychological performance and Attention-Deficit Hyperactivity Disorder subtypes and symptom dimensions. Neuropsychology, 27(1), 107-120. http://dx.doi.org/10.1037/a0030685

Norton, E., \& Wolf, M. (2012). Rapid Automatized Naming (RAN) and reading fluency: Implications for understanding and treatment of reading disabilities. Annual Review of Psychology, 63, 427-453. http://dx.doi. org/10.1146/annurev-psych-120710-100431

Pennington, B. (2006). From single to multiple deficit models of developmental disorders. Cognition, 101, 385413. http://dx.doi.org/10.1016/j.cognition.2006.04.008

Prando, M., Jacobsen, G., Moraes, A., Gonçalves, H., \& Fonseca, R. (2013). Avaliação da linguagem e do processamento auditivo na caracterização neuropsicológica do TDAH: Revisão sistemática. Psicologia em Pesquisa, 7(1), 23-36. http://dx.doi.org/10.5327/ Z19821247201300010004

Rucklidge, J.,\& Tannock, R. (2002). Neuropsychological profiles of adolescents with ADHD: Effects of reading difficulties and gender. The Journal of Child Psychology and Psychiatry, 43(8), 988-1003. http:// dx.doi.org/10.1111/1469-7610.00227

Santos, F., \& Bueno, O. (2003).Validation of the Brazilian Children's Test of Pseudoword Repetition in Portuguese speakers aged 4 to 10 years. Brazilian Journal of Medical and Biological Research, 36(11), 1533-1547. http://dx.doi. org/10.1590/S0100-879X2003001100012

Silva, J. B. L., Moura, R. J. D., Wood, G., \& Haase, V. G. (2015). Processamento fonológico e desempenho em aritmética: Uma revisão da relevância para as dificuldades de aprendizagem. Temas em Psicologia, 23(1), 157-173. doi: https://10.9788/TP2015.1-11

Snowling, M., \& Hulme, C. (2012). Annual Research Review: The nature and classification of reading disor- ders - a commentary on proposals for DSM-5. Journal of Child Psychology and Psychiatry, 53(5), 593-607. http://dx.doi.org/10.1111/j.1469-7610.2011.02495.x

Tannock, R. (2013). Rethinking ADHD and LD in DSM5: Proposed changes in diagnostic criteria. Journal of Learning Disability, 46(1), 5-25. http://dx.doi.org/10.1177/ 0022219412464341

Tannock, R., Martinussen, R., \& Frijters, J. (2000). Naming speed performance and stimulant effects indicate effortful, semantic processing deficits in Attention-Deficit/Hyperactivity Disorder. Journal of Abnormal Child Psychology, 28(3), 237-252. https:// doi.org/10.1023/a:1005192220001

Wechsler, D. (1991). Escala de Inteligência Wechsler para Crianças: Manual WISC-III ( $3^{a}$ ed). São Paulo: Casa do Psicólogo.

\section{Notas}

O presente trabalho é parte inédita da Tese de Doutorado defendida pela primeira autora, sob orientação da terceira e coorientação da segunda, no Programa de Pós-Graduação em Psicologia da Universidade Federal de Minas Gerais. Agradecimentos às escolas, profissionais da educação e da saúde, famílias e suas crianças que tornaram este trabalho possível.

Endereço para correspondência: UFRGS, Instituto de Psicologia, PPG-Psicologia, Dra. Luciana Karine de Souza, Rua Ramiro Barcelos, 2600, térreo, bairro Santa Cecília, Porto Alegre, Rio Grande do Sul, CEP: 90.035-003. Telefone: (51)3308-5246. lukarides@.gmail.com

Recebido em: 15/2/2018.

Aprovado em: 11/1/2019.

Publicado em: 11/1/2019.

\section{Marilene Tavares Cortez}

E-mail: lenacortez@gmail.com

Titulação Acadêmica: Doutorado em Psicologia. 
É MESMO (SÓ) TRANSTORNO | MARILENE TAVARES CORTEZ | LUCIANA KARINE DE SOUZA | ÂNGELA MARIA VIEIRA PINHEIRO

Afiliação Institucional: Universidade Federal de

Minas Gerais

\section{Luciana Karine de Souza.}

E-mail: lukarides@gmail.com

Titulação Acadêmica: Doutorado em Psicologia.

Afiliação Institucional: Universidade Federal de

Minas Gerais/Universidade Federal do Rio Grande

do Sul

\section{Ângela Maria Vieira Pinheiro}

E-mail:pinheiroamva@gmail.com

Titulação Acadêmica: Doutorado em Psicologia.

Afiliação Institucional: Universidade Federal de

Minas Gerais 